Cinémas

Revue d'études cinématographiques

Journal of Film Studies

\title{
La postmodernité : fragmentation des corps et synthèse des images
}

\section{Germain Lacasse}

Volume 7, numéro 1-2, automne 1996

La Représentation du corps au cinéma

URI : https://id.erudit.org/iderudit/1000938ar

DOI : https://doi.org/10.7202/1000938ar

Aller au sommaire du numéro

Éditeur(s)

Cinémas

ISSN

1181-6945 (imprimé)

1705-6500 (numérique)

Découvrir la revue

Citer cet article

Lacasse, G. (1996). La postmodernité : fragmentation des corps et synthèse des images. Cinémas, 7(1-2), 167-184. https://doi.org/10.7202/1000938ar

\section{Résumé de l'article}

Le monde de la postmodernité n'est plus conçu ni décrit comme une totalité cohérente, mais comme une complexité fragmentée. Le corps humain y est un lieu d'affrontement des techniques et de leurs représentations : dispersé par les nouvelles technologies de l'image, il est reconstruit comme centre au cinéma et simulé dans l'univers virtuel. Devant cet objet différent, le sujet ne peut plus être posté de la même façon, selon le principe de frontalité, mais plutôt pensé dans une position " variable et enveloppante » selon les termes d'Edmond Couchot. 


\title{
La postmodernité: fragmentation des corps et synthèse des images
}

\section{Germain Lacasse}

\begin{abstract}
RÉSUMÉ
Le monde de la postmodernité n'est plus conçu ni décrit comme une totalité cohérente, mais comme une complexité fragmentée. Le corps humain y est un lieu d'affrontement des techniques et de leurs représentations: dispersé par les nouvelles technologies de l'image, il est reconstruit comme centre au cinéma et simulé dans l'univers virtuel. Devant cet objet différent, le sujet ne peut plus être posté de la même façon, selon le principe de frontalité, mais plutôt pensé dans une position "variable et enveloppante" selon les termes d'Edmond Couchot.
\end{abstract}

\section{ABSTRACT}

In postmodernity, the world is no longer conceived or described as a coherent whole, but as a fragmented complexity. The human body is a site of confrontation between techniques and their representations. Dispersed by the new technologies of the visual, the body is reconstructed as centre in film, and simulated in the virtual universe. After encountering this changed object, the subject can no longer be posited in the same way, following the principle of frontality, but must be thought of as a positioning that is "variable and enveloping" in Edmond Couchot's terms.

On a parlé de postmodernisme au cinéma surtout à propos du contenu des films et de leur forme narrative: " [...] structure fragmentaire [...] multiplicité du sens [...] lecture ambiguë [...] 
esthétique du clinquant qui séduit et captive l'œil [...]" (Vanier, p. 220). On peut cependant voir ailleurs les marques du tournant postmoderne: dans les rapports entre le corps et les technologies de l'image. Il ne s'agit pas à proprement parler de cinéma, puisque je veux parler ici d'imagerie médicale et d'image de synthèse, mais il sera question de la représentation du corps sur divers écrans et de la problématique qu'elle soulève.

Jean-François Lyotard a défini la condition postmoderne comme "[...] l'incrédulité à l'égard des métarécits. Celle-ci est sans doute un effet du progrès des sciences; mais ce progrès à son tour la suppose" (Lyotard, 1979, p. 7). Le discours scientifique lui-même tend de moins en moins à s'ériger en métarécit. Lyotard reprend le discours de nombreux scientifiques modernes qui conçoivent maintenant la science comme une pensée fragmentaire plutôt que globale. Le chimiste Ilya Prigogine soulignait récemment l'importance de l'indéterminé dans les systèmes complexes, s'opposant à la conception du physicien Stephen Hawking qui croit en une théorie achevée de la physique ${ }^{\prime}$. Lyotard souligne que la science ne pourra pas davantage se désincarner que ne l'a pu le sujet de la modernité:

J'accorde à la pensée physicienne que le développement technoscientifique est l'aspect que prend présentement à la surface de la terre un processus de nég-entropie ou de complexification qui est en cours depuis qu'il y a la terre. [...] J'accorde que l'intelligence sans corps que tout ici travaille à créer permettra de relever le défi opposé au processus de complexification par le raz de marée entropique en quoi consiste, de ce point de vue, l'explosion solaire à venir. [...] Le corps humain, comme ensemble matériel, gêne la séparabilité de cette intelligence, son exil, et donc sa survie. Mais le corps, phénoménologique, mortel, percevant, est en même temps le seul analogon disponible pour penser une certaine complexité de la pensée (Lyotard, 1988, p. 30).

Je crois que l'on peut démontrer ce qu'avance Lyotard en comparant les deux utilisations mentionnées des technologies de l'image: la première, qui fragmente le corps pour le livrer au savoir médical positiviste et systématique, la seconde, qui essaie de réintroduire le corps et la sensibilité dans les machines à penser. 


\section{Fragmentation des corps}

On parle depuis quelque temps de pénétrer l'image, surtout quand on traite de télévision interactive ou de réalité virtuelle. Pourtant, d'une certaine façon, c'est aussi l'image qui pénètre le corps, les caméras y étant maintenant introduites pour en faire voir l'intérieur. Cette expérience n'est d'ailleurs pas nouvelle: les rayons $\mathrm{X}$ furent inventés en même temps que le cinéma et exploités d'abord comme un spectacle: "Entrez voir les os de votre main!" affichait-on à la porte de certaines baraques foraines en 1900. Notre arrière-grand-père entrait voir ce dont il avait l'air, tel que filmé par un cinématographe, et pouvait de l'autre côté de la rue aller voir son squelette; ses arrière-petits-enfants peuvent aujourd'hui regarder sur un téléviseur leur apparence à l'état fotal, l'échographie ayant été enregistrée sur ruban magnétique.

La caméra pénètre donc maintenant le corps. Le tomographe (scanner) et l'échographe le reproduisent sur deux ou trois plans. Les endoscopes sont introduits dans l'organisme pour visualiser les organes, les lésions et l'outil d'intervention. Le Voyage fantastique, film produit il y a 30 ans (R. Fleischer, 1966) et mettant en vedette Raquel Welch, racontait le voyage d'un sous-marin et de son équipage qu'on avait miniaturisés pour aller effectuer une intervention chirurgicale dans le corps d'un savant. On pourrait aujourd'hui en faire un remake en se servant des images d'un endoscope comme décor.

Maintenant, le rêve est poussé bien plus loin: Wim Wenders, dans Jusqu'au bout du monde (1991), fait inventer une caméra vidéo capable de filmer les rêves. Il est néanmoins en retard: les outils pour explorer l'inconscient et l'imaginaire sont probablement aussi anciens que le couteau de silex du premier chirurgien. Les incantations des sorciers-guérisseurs sont apparues il y a quelques millions d'années, peut-être en même temps que les premières trépanations. La technologie qui montre les viscères du sujet est nettement plus récente. Maintenant, des instruments pénètrent même le corps pour y déposer d'autres instruments, substituts d'un organisme défaillant, l'exemple le plus simple étant le stimulateur cardiaque. Les étudiants en médecine utiliseront bientôt des images de synthèse, même si leurs patients ont parfois des corps semi-synthétiques! 


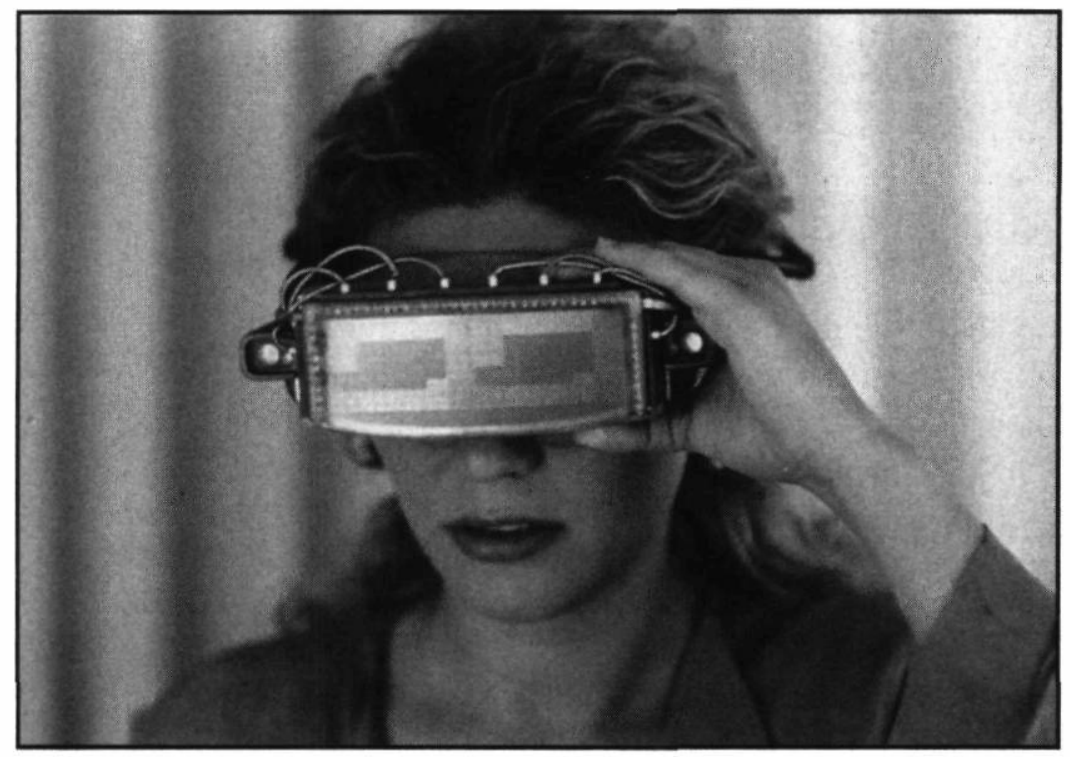

Jusqu'au bout du monde de Wim Wenders (1991) Collection Cinémathèque québécoise

Le gouvernement américain investit actuellement des sommes importantes ( 25 millions de dollars jusqu'à maintenant) dans des projets de "cartographie du cerveau"; on veut ni plus ni moins que la réalisation d'un atlas informatisé du cerveau: Human Brain Mapping Project. L'Institut neurologique de Montréal est d'ailleurs un des centres de recherche participant à ce projet, par l'intermédiaire du Centre McConnell d'imagerie du cerveau. Un de ses chercheurs expliquait récemment la complexité du problème et la puissance informatique nécessaire: le cerveau compte 10 milliards de neurones et 160000 kilomètres de fibre nerveuse ${ }^{2}$.

La cartographie du cerveau reposera sur les principales techniques d'imagerie médicale: imagerie à résonance magnétique (IRM), tomographie par émission de positrons (TEP), tomographie axiale, DSA pour les vaisseaux sanguins, et même une "antiquité " comme l'électro-encéphalogramme. Les techniques anciennes (plus de 10 ans!) comme la TEP faisaient appel à un traceur radioactif dont on observait sur écran la pénétration dans les organes. Cette méthode est déjà dépassée: l'IRM force 
l'alignement des atomes d'hydrogène présents dans les tissus par la force d'un champ magnétique, puis émet un signal radio qui perturbe l'alignement. Quand cesse l'émission, les atomes retrouvent leur place dans l'alignement et l'ordinateur enregistre ces mouvements pour constituer une carte de la zone explorée.

Cette technique rappelle une des premières fonctions des cinématographes, notée par Paul Virilio: montée sur les premiers avions de bombardement, la caméra permettait de tenir les cartes militaires à jour en montrant les cratères creusés par les bombes ou les édifices détruits (Virilio, 1980). En fait, on n'est plus très loin de cette idée de Wenders: on pourra bientôt voir ce dont les gens rêvent. Le patient s'étendra sur un divan et sur l'écran du scanner, le psychiatre regardera son rêve. Neuropsychologues et spécialistes de la cognition nous proposeront bientôt des modèles...

Michel Foucault s'est intéressé, dans Surveiller et punir. Naissance de la prison, au "panoptisme" ou panopticon, une prison imaginée par Jeremy Bentham comme une structure architecturale dessinée autour d'un point central d'où il serait possible de tout voir en permanence, où le surveillant verrait tout et le surveillé sentirait constamment l'œil du gardien. Au lieu d'un système de présence réelle de l'autorité, il s'agit pour Foucault d'un système oppressif dont la présence réside dans le contrôle total et les effets qu'il a sur les sujets sociaux. On pourrait penser que le projecteur de cinéma et ensuite la télévision ont joué ce rôle, mais ce serait trop de méchanceté...

Par contre, l'imagerie médicale correspond assez bien à ce que pensait Foucault. Il écrivait que la visibilité du monarque a été remplacée par la visibilité permanente du sujet, de tous les sujets; au lieu des rituels brutaux qui manifestaient l'ancien pouvoir (torture, exécutions publiques, etc.) sont apparus de nouveaux rituels, produits d'un pouvoir diffus dans tout le corps social et assurant la présence de la norme. "One such ritual form is the examination which assumes a central place in this new technology of subjectification ", écrit Roy Boyne en commentant Foucault (1990, p. 113).

Les techniques d'imagerie médicale sont aussi devenues l'indispensable outil ou complément des techniques de procréation assis- 
tée, et ont contribué à l'élaboration de tout le discours de légitimation de ces techniques. Sarah Franklin pense que le film de type docu-drame The World of the Unborn (Panorama, 1988) force le spectateur à adopter le point de vue du médecin ou du technicien :

A sense of bodily permeability is created, as the camera moves seamlessly from the outside to the inside of the woman's body. Technological powers of magnification and penetration privilege the viewer's gaze, "just as they do to the clinicians'." The spectator position is established through the clinical gaze, as the camera moves down the laparoscope. The viewpoint is thus not only that of the clinicians, it is through their technology, literally through their lenses, which, in this case, are multiple (Franklin, p. 537).

Franklin souligne aussi le fait que la nature et la technologie semblent se fondre, ne faire qu'une. On assiste à la création et à la légitimation d'un nouveau récit, où la procréation n'est plus une question d'abeilles et de chou, de "mystère de la nature", de relation entre hommes et femmes, mais où l'intervention du médecin et du technicien semble aller de soi, faire partie de la nature des choses. Ce nouvel épisode du métarécit scientifique a cependant été assez rapidement confronté à une autre narration, celle des femmes dont le corps se retrouve sur l'écran. La critique féministe a été la plus vigilante et la plus perspicace face aux techniques d'imagerie médicale, sans doute parce que le corps de la femme a été un des premiers lieux d'expérimentation de cet outillage. Ella Shohat a montré comment l'endométriose, problème essentiellement féminin lié à la fertilité, n'a été traitée que très superficiellement par les médecins employant la laparoscopie. On a construit tout un discours de légitimation de cette technologie, mais en réalité elle n'aurait presque rien changé au problème sinon amené une façon différente d'en atténuer les complications.

Le nouvel outillage n'a bien servi les femmes qu'à partir du moment où elles ont formé des groupes d'entraide qui ont utilisé la laparoscopie pour comprendre leur propre corps et étudier la prévention, au lieu de livrer le corps au regard et au pouvoir du médecin : 
Working closely with the Endometriosis Association, doctors such as Maria Penner Perper and Camran Nezhat, who conceive of making the "doctor-patient relationship truly a partnership, " disrupt science's institutional authority. These few pockets of disruption represent the possibility of shifting from a science constituted by knowledge and power to a science shaped by community values and needs (Shohat, p. 79).

Ella Shohat parle justement d'holisme et de prévention par opposition au traitement uniquement technologique qui profite surtout aux fabricants de machineries de vision (et de division).

L'imagerie médicale a aussi créé une sorte de nouvelle spectature, celle d'une personne qui peut regarder l'intérieur de son propre corps (Shohat appelle cela self-spectatorship), mais aussi une nouvelle narration, un nouveau récit, celui de la transformation de l'intérieur du corps par la science. Si ce récit a été encadré dans une démarche de prévention et de contrôle de la femme sur son corps et sur l'intervention, le fait de voir l'image de l'intervention est positif, lui donne l'impression d'un plus grand pouvoir sur son corps. Sans cela, c'est le point de vue du chirurgien qui prévaut, parce que la caméra épouse son point de vue; le visionnement est alors souvent refusé par les patientes, ou accepté mais avec appréhension et peut s'avérer traumatisant.

Ces images nous ramènent assez loin dans le passé, à René Descartes en fait: ne concevait-il pas le corps comme une machine, un assemblage de pièces? Rudolf Zur Lippe, rappelant ces conceptions de Descartes, en voit la suite chez Adam Smith et Kant et pense qu'il faudra attendre jusqu'à Nietzsche pour voir réapparaître une pensée protestant contre le morcellement du corps. Zur Lippe écrit que chez les Grecs de la période classique, la notion de corps n'existe pas:

Quelqu'un qui se lave, qui fait couler de l'eau sur son corps, fait couler cette eau sur le kros, ce qui enveloppe le corps. Cependant, il ne s'agit pas d'atomiser, de morceler le corps; à l'époque d'Homère, l'unité du corps est justement constituée par la multiplicité de ses aspects; c'est à la condition de passer à travers cet apparent paradoxe que la compréhension du corps peut être approfondie (p. 29). 
Zur Lippe pense que le corps ne devient fiction que si on sépare le physique et le psychique d'une part, et qu'on le sépare du monde dans lequel nous vivons pour le replacer face à lui plutôt qu'en lui.

Le corps a été fictionnalisé en étant fragmenté et ce processus commence grosso modo à la Renaissance, en 1482 plus précisément, date de la première reproduction iconographique d'une dissection dans un livre imprimé. "La chirurgie est donc grammaire autant que graphique, commente Philippe Quéau. Le tracé du scalpel obéit au savoir anatomique qui est lui-même lié au découpage linguistique. On voit le corps comme on le nomme" (1986, p. 73). Ou bien on le nomme comme on le voit, car c'est aussi le début de la peinture perspectiviste: ainsi les viscères commencent à être reproduites au moment où le sujet est constitué dans la peinture. La pénétration du corps par ces techniques d'image ne découle-t-elle pas du même vieux rêve platonicien de découverte de la vérité, d'une vérité scientifique, ou du rêve de Wittgenstein d'un langage réfléchissant parfaitement la réalité du monde? L'endoscopie et la tomographie sontelles la vérité du corps ou celle du savoir médical?

Si l'image pénètre le corps et le rend comme transparent, réfléchit-elle néanmoins "exactement" l'intérieur du corps, n'est-elle pas produite au contraire par une technique qui est une volonté, un miroir orienté et déformant? Ces images du corps ne sontelles pas plus proches de la vérité puisqu'elles nous mettent en présence de la maladie, de la tumeur à l'origine de la maladie? Ce corps, le nôtre, projeté sur l'écran du tomographe ou de l'endoscope, le percevons-nous mieux ainsi qu'avec nos autres sens? Cette question nous ramène à un débat vieux comme la philosophie, celui de la distance entre le signifiant et le signifié, auquel Jacques Derrida a proposé une réponse différente qui s'appliquerait peut-être bien ici : tout langage est une représentation, la recherche d'une origine est une mystification justifiant tous les substituts d'une introuvable vérité. "Le signe et la divinité ont le même lieu et le même temps de naissance. L'époque du signe est essentiellement théologique" (Derrida, p. 25). Cette image à l'écran est le signe dont le médecin est le grand-prêtre. Donnant raison à Lyotard, c'est une des dernières icônes de 
l'époque des métarécits, de la modernité dont le discours a atomisé le corps pour en nommer et étudier chaque fragment, mais en oubliant le plus souvent qu'il fait partie d'un tout.

\section{Synthèse des images}

La synthèse d'image, l'image créée par ordinateur, pourrait être qualifiée de technologie postmoderne au sens de Lyotard dans la mesure où elle peut devenir le lieu de microrécits où le corps semble vouloir retrouver ses droits dans un univers qui était au départ purement rationnel et cérébral.

La déception procurée par ces organes de pensée sans corps, écrit Lyotard, provient de ce qu'ils opèrent en logique binaire, celle qui s'est imposée avec la logique mathématique de Russell et Whitehead, la machine de Turing, le modèle neuronal de McCulloch et Pitts, la cybernétique de Wiener et von Neumann, l'algèbre de Boole, l'informatique de Shannon. Or, objecte Dreyfus, la pensée humaine ne pense pas en binaire. Elle ne travaille pas sur des unités d'informations (les bits) mais sur des configurations intuitives et hypothétiques. [...] Or, telles sont les opérations paradoxales qui constituent l'expérience du corps, du corps dit "propre", phénoménologique, dans son espace-temps de sensibilité et de perception. Et c'est pourquoi il conviendrait de le prendre en modèle pour la fabrication et la programmation des intelligences artificielles si l'on entend que celles-ci ne se bornent pas à la faculté de raisonner logiquement (1988, p. 23).

Le vœu de Lyotard semble avoir inspiré plusieurs des premiers artistes de l'image de synthèse, qui se sont très vite lancés dans la simulation du corps à l'écran. Rebecca Allen, chercheuse américaine engagée depuis longtemps dans l'animation par ordinateur, utilise la machine pour animer des images tridimensionnelles de danseurs, et souligne les possibilités de conscience différente qu'amène le virtuel: "In this piece, computers can be seen as extensions of ourselves, capable of imitating what we teach them. In turn, by observing and learning from our new, synthetic reflexion, we should be better able to understand and move closer to our ideals" (Allen, p. 331). 
Anne-Marie Duguet pense que la vidéo s'est approprié les corps, mais que le virtuel produit des corps auxquels il reste à procurer une âme :

\begin{abstract}
L'animation ne suffit pas à "donner vie " à ces androïdes, il leur manque encore l'expression. Alors pourquoi ne pas procéder à des prélèvements d'âme chez l'humain et les conférer au corps virtuel, comme on pratique les hybridations d'abricot-pêche ou de pommepoire? C'est par une manipulation génétique de cette sorte qu'a été créé le pianiste Tony de Peltrie (p. 79).
\end{abstract}

Les images de synthèse ne datent pas d'hier; elles font même partie désormais de l'arsenal d'ébahissement des films les plus coûteux, ces métarécits... Terminator II (1992) a fasciné le public avec son robot caméléon qui prenait la couleur et la forme du milieu. Plus récemment, Jurassic Park (1993) a attiré encore plus de gens et d'argent avec ses dinosaures synthétiques en partie made in Québec. Mais cela a commencé il y a déjà plus d'une dizaine d'années. Et dans les Cahiers du cinéma, en 1987, Thierry Cazals écrivait un article très bien documenté sur les "nouvelles images", intitulé "Le monde comme simulacre et programmation". Il pouvait déjà y décrire et comparer plusieurs petits films faits en animation par ordinateur. Dans ces descriptions, Cazals se montrait cependant fort soupçonneux :

La synthèse d'image est à la fois déréalisation du monde (réduit à des séquences d'informations chiffrées, des modèles algorithmiques) et recréation artificielle de l'effet de réel. Désormais, elle n'est plus un simple jeu de Lego géométrique (sphères, plans, volumes élémentaires) mais aussi une reformulation du monde perceptible (p. 52).

La métaphore corporelle (et médicale) est très souvent utilisée pour parler de l'image de synthèse. Ainsi Cazals écrit que «[...] la greffe d'images synthétiques équivaut à une sorte de cancer: la perversion cellule après cellule, plan après plan, de tout le tissu filmique» (p. 59). Cazals énumère les premiers films de synthèse français; à propos de l'Unique de Jérôme DiamantBerger (1985), il parle de "[...] chanteuse dépossédée de son 
corps... rien d'autre qu'une actrice supplantée par son image". Quant à Terminus de Pierre Jenn (1986), il le qualifie de "[...] symptomatique de cette contagion/substitution". Il écrit encore que "[...] le cinéma passe par des acteurs, des médiums vivants et pas par des marionnettes ou des clones numérisés "; au sujet du cinéaste Yoichiro Kawaguchi, il écrit que celui-ci explore "[...] un univers très matriciel, utérin. [...] Avec l'ordinateur, l'œil peut se séparer du corps et se tourner vers le dedans des choses, au cœur même des programmes ".

Thierry Cazals se montre fort soupçonneux face à ces images "qui savent que nous les regardons", selon l'expression d'Edmond Couchot. D'autres se méprennent au point de penser que la simulation est le début de la confusion du réel et du modèle. Jean Baudrillard écrit :

\begin{abstract}
Il n'y a plus de distance critique et spéculative du réel au rationnel. Il n'y a même plus exactement projection de modèles dans le réel (ce qui équivaut encore à la substitution de la carte au territoire chez Borges), mais transfiguration sur place, ici et maintenant, du réel en modèle. Court-circuit fantastique: le réel est hyperréalisé. L'hyperréel est l'abolition du réel non par destruction violente, mais par assomption, élévation à la puissance du modèle. Anticipation, dissuasion, transfiguration préventive, etc. : le modèle opère comme sphère d'absorption du réel (1982, p. 114).
\end{abstract}

Le même Baudrillard, pendant la guerre en Irak, s'est mépris au point d'affirmer qu'il s'agissait d'une guerre simulée, un spectacle de guerre, oubliant que les images simulées de la chaîne de télévision CNN avaient surtout servi à masquer les milliers de victimes irakiennes (Baudrillard, cité dans Norris, 1992).

Si on comprenait mieux la nature des nouvelles réalités que sont les images synthétiques et le cyberespace, on éviterait sans doute de telles méprises. Ses théoriciens pensent que ce nouveau réel n'a pas que l'apparence de la réalité, n'est pas qu'un simulacre, mais qu'il a ses constituants spécifiques. Ces images sont le produit de modèles conçus à l'aide de langages formalisés, euxmêmes inspirés des sciences mathématiques ou physiques. Ces langages ont atteint un degré d'abstraction extrêmement élevé, 
au point que le théoricien Timothy Binkley croit qu'ils peuvent transformer des séquences de nombres en faits perceptibles:

So the process by which a digital image gets made is represented by a function that looks something more like: $\mathrm{p}=\mathrm{M}(\mathrm{C}(\mathrm{n})$ ), when $\mathrm{n}$ is a number (or perhaps a set of numbers) that is subjected to calculations, C, whose results are subsequently materialised through an interface, $M$, which turns numbers into perceivable events (p. 115).

Plusieurs pensent que ces images ne sont pas des simulations, mais un réel autonome, créé de toutes pièces. Edmond Couchot, praticien et théoricien de l'image de synthèse, écrit:

À la logique de la Représentation optique succède la logique de la Simulation numérique. Cette recréation du réel n'est évidemment qu'approchée, mais la logique de la simulation tend à l'accomplir avec le maximum de précision, selon des lois rationnelles qui le décrivent ou l'expliquent. Elle cherche à recréer de toutes pièces une réalité autonome, avec toute sa profondeur structurelle et fonctionnelle (1991, p. 17).

Couchot dit encore autre chose, quelque chose de capital pour le propos que nous tenons ici :

Le sujet interactif ne s'éprouve plus en tant que sujet (et individu: entité non divisible) dans un rapport frontal à l'objet et à l'image qui enregistre la trace de cette confrontation, mais dans une saisie holistique, enveloppante, qui prend prise en chacun des points de la matrice constituant le modèle numérique de l'objet $\left(1987\right.$, p. $\left.93^{3}\right)$.

Holisme: le même terme était employé par Ella Shohat pour parler d'une utilisation différente des images médicales. On le retrouve ailleurs aussi, évidemment beaucoup en médecine "alternative", mais également dans les textes relatifs à la nouvelle épistémologie scientifique, appelée également science postmoderne :

Against the mechanism, reductionism, universalism and dualism that characterize much modern thought, 
postmodern emphases on evolution, difference, heterogeneity, indeterminacy and holism have important consequences for the way we think about nature and orientate ourselves to it (Best, 1991, p. 222 ${ }^{4}$ ).

Le paradigme holistique est intéressant parce qu'il peut fort bien s'appliquer à la représentation du corps dans l'univers virtuel, où on semble vouloir recréer l'intégrité de l'expérience sensible. Tandis que Baudrillard parle de "lieu de disparition du sens", certains cherchent les paramètres qui permettraient de faire ce à quoi appelle Lyotard: recréer la sensibilité humaine dans le cyberespace:

We need to study those elements of time and communication in cyberspace that have created the vernacular architecture of virtuality. We need to recognize the meaning of presence in cyberspace and the manners in which its raw materials can be used to form orders from which we draw the very basic needs of security and confidence. And from the awareness of meaningful presence we will be able to bring beauty, pleasure or whatever other emotional relationships and echoes we want (Ludvigsen, 1993, p. 5).

\section{Un sujet intègre dans un univers éclaté}

La postmodernité signale peut-être le retour du corps comme mémoire; le rationalisme moderniste voulait s'affranchir de la tradition, c'était la volonté des Lumières (Kant), mais elle oubliait le corps, cette matérialité issue du monde et qui "donne corps" à notre rationalité. "Le corps est névrotique parce que son histoire est hétéronome, victime d'habitudes et névroses "inhumaines" qui empêchent l'autonomie du sujet" ". Le corps est comme le monde, il échappe constamment à l'appréhension par le savoir et le regard. C'est pour cela que Michel Foucault s'en est pris à la médicalisation; il a mené une croisade contre l'enfermement du corps et de l'esprit non pour condamner la médecine, mais pour en montrer la prétention et les limites. Il ne faudrait peut-être plus essayer de trouver des théories qui expliquent le monde et le corps, mais seulement des concepts pour se maintenir à leur écoute. Des historiens de la médecine 
soutiennent maintenant les vues de Foucault, du moins ceux qui admettent le relativisme du savoir médical ${ }^{6}$. Pour Jean-Charles Sournia par exemple, la médecine scientifique n'est qu'un des discours sur le corps, ce n'est qu'une des relations possibles au corps. Quant aux images, médicales ou autres, on veut maintenant les voir avec tout le corps.

Le corps qu'on peut maintenant synthétiser à l'écran, il faut donc aussi apprendre à le synthétiser devant l'écran et qu'au lieu de deux robots humanoïdes qui se font face de part et d'autre de l'écran, il y ait d'un côté un homme ou une femme, de l'autre une image, robotique ou autre. Certains parlent de symbiose de l'humain et du machinique et ils n'ont pas tort, disant que la fiction virtuelle correspond à un désir profond et qu'elle est comme tatouée sur nos corps. "La fiction technologique s'inscrit dans et sur notre chair. Une phénoménologie nouvelle est à construire" écrit Ollivier Dyens (p. 31). La fiction s'inscrit sur notre corps, mais celui-ci pénètre aussi dans la fiction qu'il a créée. Pensons à une autre fiction: une personne manipule un logiciel permettant d'animer à l'écran un personnage virtuel. Mais cette personne a un bras "artificiel", entend grâce à un implant cochléaire et survit grâce à un stimulateur cardiaque devant cet écran où s'agite un personnage avec simulateur cardiaque.

Il ne s'agit que d'une fiction, mais ces deux personnages qui se font face sont tous deux mi-humains et mi-machiniques, le personnage virtuel n'étant qu'une prothèse plus complète. L'écran qui sépare ces deux univers s'amincit de plus en plus parce que de chaque côté, ces deux personnages essaient de le traverser; ils n'y parviendront peut-être jamais (peut-être), mais ils vivront de plus en plus près de la surface de cet écran. Certains refusent cette trajectoire, agitent leur corps pour occuper l'espace réel et empêcher d'être happés par l'autre côté ou se trouver trop près de la surface. Pierre Hébert, cinéaste d'animation, s'inquiète de "l'effacement du corps" que supposent les nouvelles techniques (Hébert, 1989). Pourtant, s'il veut préserver l'intégrité et la totalité de l'expérience sensorielle du créateur dans la création, il oublie que le spectateur veut en faire autant: c'est probablement pour cette raison qu'on essaie de créer ces 
jeux virtuels où le joueur pourrait explorer un univers virtuel en utilisant tout son corps.

Il faut peut-être lire plutôt dans ces nouvelles structures un sens différent; la multiplicité du sens constatée dans les œuvres postmodernes, ce peut être aussi bien un retour à la multiplicité sensorielle, après des siècles de rationalisme et des périodes de spécialisations orale et visuelle. Fernande St-Martin soulignait récemment que depuis fort longtemps, la rationalité semble associée à la linéarité et à la temporalité, tandis que l'espace est devenu le territoire de l'inconscient, et que les philosophes ne font que commencer à revenir vers la sensation et l'espace, auxquels les artistes visuels s'intéressent depuis bien plus longtemps:

Seraient encore justes aujourd'hui les observations de Freud, en 1911, selon lesquelles la raison, ou la pensée occidentale, s'est tout entière inscrite dans la structure vectorielle et successive de la temporalité, alors que l'espace a été relégué aux zones obscures de l'inconscient (St-Martin, p. 241).

La pensée contemporaine commence, je crois, a réinvestir l'espace et cela se fait curieusement à partir du moment où elle accepte un rôle plus modeste. La pensée n'est pas tant fragmentée que réduite à des objectifs plus modestes, mise en doute dans ses visées globales et totalisantes (ou totalitaires!), c'est d'ailleurs l'essentiel de ce que Lyotard a appelé la condition postmoderne. Le monde est fragmenté, complexe, mais la pensée se refuse dorénavant à vouloir lui fournir une cohérence qu'il n'a peutêtre jamais eue. Si cohérence il y a, elle sera fluide plutôt que dure, le savoir ne sera plus une superstructure d'acier (la tour Eiffel) mais une onde fluctuante. S'il faut un monument pour la modernité, il devrait être ainsi: une base en poutrelles d'acier sur laquelle reposerait un écran cathodique où un personnage se transformerait constamment en chacun de ses organes sensitifs.

René Payant, qui fut professeur à l'Université de Montréal et mourut très jeune, eut le temps de faire quelques réflexions importantes: "La condition postmoderne, c'est un devoirinventer un nouvel ordre de choses et une nouvelle conception de l'ordre qui ne craint pas de remplacer l'idée de cohérence sans faille par celle de complexité", comme le pense Henri Atlan 
(p. 241). Cette pensée d'une hétérogénéité du monde n'est pas incompatible avec l'hétéronomie du corps, elle est peut-être sa condition, et on peut y voir un lien avec le fait que les théoriciens des nouvelles sciences et des nouvelles images parlent d'holisme, comme on en parle en médecine. La nouvelle position du sujet dont parle Couchot est peut-être aussi visible dans le rapport du spectateur à certains films. À propos de son film Prospero's Books (1992), Peter Greenaway disait qu'il voulait en préparer une version sur disque au laser, version "interactive " permettant au spectateur "[...] d'en décortiquer librement les éléments" (Privet, p. 13). Au lieu d'un spectateur dominé par un film qui le captive et le subjugue, un spectateur fragmenté par une œuvre qu'il ne perçoit qu'avec ses yeux, nous aurions un spectateur qui dissèque le film. Ces expériences de film interactif pourraient aussi être comparées aux jeux de fiction interactive par ordinateur, les "MUD» (Multi User Display) du réseau Internet, où des usagers créent un personnage qu'ils pilotent à leur gré dans un récit "collectif".

Ce serait ainsi au moment où il peut faire corps à corps avec son double écranique que le sujet moderne s'extrairait enfin de la spectature. Au lieu de voir son corps disséqué par le regard omniscient de la rationalité cartésienne qui voulait cerner le monde par l'esprit, il retrouverait une intégralité sensitive qui le ramènerait au centre d'un monde qu'il peut explorer de multiples façons. On peut rêver: la fin de la division des sens serait peut-être aussi la fin de la division sociale. Il y a une condition essentielle: cette unité doit se faire devant l'écran, non dans l'univers virtuel; mais celui-ci pourrait y contribuer.

Université de Montréal

\section{NOTES}

1 Voir à ce sujet André Lemelin, "L'univers, le temps et la liberté. La conception d'Ilya Prigogine ", Interface (janvier-février 1994).

2 Pour quelques détails, voir Benoit Munger, "Les géographes du cerveau ", Le Devoir, 15 décembre 1993, p. B 1.

3 Souligné par nous.

4 Souligné par nous. 
5 Propos tirés d'une conférence qu'a donnée Bill Readings à l'Université de Montréal, le 12 mai 1994, intitulée Postmodernité / Postmodernisme.

6 L'historien de la médecine Jean-Charles Sournia écrivait récemment : "De l’ignorance, nous serions passés à la connaissance par illuminations successives. Même si l'idée de cette progression assez soudaine reste respectable, on ne peut pour autant nier la valeur de méthodes plus primitives, abandonnées parce que dérisoires à nos yeux, alors que d'autres populations africaines ou andines les conservent. Elles représentent certainement les premiers stades embryonnaires d'une médecine qui deviendra rigoureuse, mais elles répondent néanmoins à une permanence de l'esprit humain et à une constante toujours actuelle de ses comportements" (Histoire de la médecine, Paris: Éditions La Découverte, 1992, p. 182).

\section{OUVRAGES CITÉS}

Allen, Rebecca. "Computer Animation: A New Aesthetic Approach ", L'Imaginaire numérique. Actes du colloque interdisciplinaire. Paris-Londres-Lausanne: Éditions Hermès, 1986.

Baudrillard, Jean. À l'ombre des majorités silencieuses. Paris : Denoël-Gonthier, 1982.

Best, Steven. "Chaos and Entropy. Metaphors in Postmodern Science and Social Theory". Science as Culture, vol. 2, n" 11 (1991).

Binkley, Timothy. "Refiguring Culture", dans Phillip Hayward et Tana Wollen (direction), Future Visions. New Technologies of the Screen. London: British Film Institute, 1993.

Boyne, Roy. Foucault and Derrida. The Other Side of Reason. London: UnwinHyman, 1990.

Cazals, Thierry. "Le monde comme simulacre et programmation ", Cahiers $d u$ cinéma, n" 399 (1987).

Couchot, Edmond. "Un fracassant Big Bang", Cinémas, vol. 1, n” 3 (1992).

Couchot, Edmond. "Sujet, objet, image", Cahiers internationaux de sociologie, vol. LXXXII (1987).

Derrida, Jacques. De la Grammatologie. Paris : Éditions de Minuit, 1970.

Duguet, Anne-Marie. "Le double hérétique». Paysages virtuels, image vidéo, image de synthèse. Paris: Editions Dis Voir, 1988.

Dyens, Ollivier. "Art et cyberespace: la technologie du sensible». Dire, vol. 3, n" 3 (1994).

Franklin, Sarah. "Postmodern Procreation. Representing Reproductive Practice». Science as Culture, vol. 3, nº 17 (1993).

Hébert, Pierre. "Les enjeux de l'art à l'ère des machines. Effacement et résistance du corps". 24 Images, n" 43 (1989).

Lemelin, André. "L'univers, le temps et la liberté. La conception d'Ilya Prigogine». Interface (janvier-février 1994).

Ludvigsen, Borre. "Presence and Form in the Architecture of Cyberspace". The Architecture of Cyberspace. Proceedings of INET 1993.

Lyotard, Jean-François. "Si l'on peut penser sans corps", L'Inhumain. Causeries sur le temps. Paris: Galilée, 1988.

Lyotard, Jean-François. La Condition post-moderne. Paris: Minuit, 1979.

Munger, Benoit. "Les géographes du cerveau ». Le Devoir, 15 décembre 1993.

Norris, Christopher. Uncritical Theory. Postmodernism, Intellectuals, and the Gulf War. Boston, University of Massachusetts Press, 1992. 
Payant, René. "Le postmodernisme selon le cinéma", dans Michel Larouche (direction), Le Cinéma aujourd'hui. Films, théories, nouvelles approches. Montréal : Guernica, 1988.

Privet, Georges. «Peter Greenaway, l'oiseau rare». Voir, semaine du 15 au 21 octobre 1992.

Quéau, Philippe. Éloge de la simulation. De la vie des langages à la synthèse des images. Paris: Champ Vallon, 1986.

Shohat, Ella. "Lasers for Ladies: Endo Discourse and the Inscriptions of Science". Camera Obscura, n' 29 (1993).

Sournia, Jean-Charles. Histoire de la médecine. Paris: Éditions La Découverte, 1992.

St-Martin, Fernande. "Sémiologie visuelle et philosophie du langage", dans Marie Carani (direction), De l'histoire de l'art à la sémiotique visuelle. Québec: Éditions du Septentrion, 1992.

Vanier, Hélène. "L'allégorie chez Ruiz ", dans Michel Larouche (direction), Le Cinéma aujourd'hui. Films, théories, nouvelles approches. Montréal: Guernica, 1988.

Virilio, Paul. Esthétique de la disparition. Paris: Balland, 1980.

Zur Lippe, Rudolf. "Une unité problématique: éléments pour une histoire des conceptions du corps", dans Claude Reichler (direction), Le Corps et ses fictions. Paris: Minuit, 1983. 